\title{
Quantitative is better than qualitative
}

Participants in cancer screening programs who receive a negative result on the first test often consider themselves to be at low risk and do not return for further screening. This obviously means that it is crucial to ensure that the test is as accurate as possible on the first use and to assign people with a negative result into risk groups to encourage them to return for future tests.

An example of such a screen is the immunochemical fecal occult blood test (iFOBT) that tests for colorectal cancer. Researchers in Taiwan, led by Hsiu-Hsi Chen, conducted a prospective study using the iFOBT test with a cutoff fecal hemoglobin level of $100 \mathrm{ng} / \mathrm{ml}$. In all, 44,324 participants with a baseline level of below the cutoff (negative) and 1,668 above the cutoff (positive) were followed up for a median of 4.39 years.

The investigators observed that 'negative' participants with a baseline concentration of $1-19 \mathrm{ng} / \mathrm{ml}$ had a colorectal cancer incidence of 1.74 per 1,000 person-years, which increased to
7.08 per 1,000 person-years in those with a baseline concentration of $80-99 \mathrm{ng} / \mathrm{ml}$. "Baseline fecal hemoglobin concentration can be predictive of incident colorectal neoplasia among negative screenees at first screen in a concentration-dependent manner," explains Chen. "We are tempted to develop a risk prediction model, with the incorporation of demographic characteristics, baseline fecal hemoglobin, family history and dietary factors, to facilitate individual-tailored screening." It remains to be seen if such a model combining quantitative iFOBT with other risk factors would encourage people to return for future screening. But, these results strongly indicate that a quantitative test could improve screening reliability.

\section{Rebecca Kirk}

Original article Chen, L. S. et al. Baseline faecal occult blood concentration as a predictor of incident colorectal neoplasia: longitudinal follow-up of a Taiwanese population-based colorectal cancer screening cohort. Lancet Oncol. 6, 551-558 (2011) 